\title{
Upregulation of microRNA-132 in gastric cancer promotes cell proliferation via retinoblastoma 1 targeting
}

\author{
FENG-YU GAO ${ }^{1}$, QUN-YING LIU ${ }^{2}$, LI YUAN $^{3}$ and SHI-YING XUAN ${ }^{4}$ \\ ${ }^{1}$ Department of Gastroenterology, Hiser Medical Group of Qingdao, Medical College of Qingdao University, Qingdao, \\ Shandong 266033; ${ }^{2}$ The Maternity Centre, The No. 8 People's Hospital of Qingdao; ${ }^{3}$ Department of Rehabilitation, \\ The Third People's Hospital of Qingdao, Qingdao, Shandong 266041; ${ }^{4}$ Department of Gastroenterology, \\ Qingdao Municipal Hospital, Medical College of Qingdao University, Qingdao, Shandong 266033, P.R. China
}

Received October 23, 2014; Accepted June 18, 2015

DOI: $10.3892 / \mathrm{mmr} .2015 .4253$

\begin{abstract}
Gastric cancer is one of the most frequent malignancies and a leading cause of cancer-related mortality worldwide. MicroRNAs (miRs), a class of small non-coding RNAs, have been shown to be critical in tumorigenesis. In the present study, the expression levels of miR-132 were analyzed in gastric cancer samples using quantitative reverse transcription-polymerase chain reaction. In addition, the cell viability, proliferation and invasion abilities were determined in two gastric cancer cell lines, NCI-N87 and MGC80-3, that were transfected with miR-132 mimics or antisense oligos. It was found that miR-132 expression was significantly upregulated in gastric cancer tissues when compared with adjacent non-cancerous tissues. At the molecular level, the data demonstrated that miR-132 inhibits the protein levels of retinoblastoma 1 (RB1) by targeting the 3'-untranslated region. Furthermore, reintroduction of RB1 markedly attenuated the proliferative roles of miR-132 overexpression. Therefore, the present results indicate that the miR-132/RB1 regulatory axis may be a potential novel diagnostic and therapeutic target for the treatment of gastric cancer.
\end{abstract}

\section{Introduction}

Gastric cancer has become one of the leading causes of cancer-related mortality in Asia (1). Therefore, an improved understanding of its pathogenic factors and molecular mechanisms may facilitate the identification of precise prognostic markers and effective therapeutic targets.

MicroRNAs (miRs), a class of small non-coding RNAs of $\sim 22$ nucleotides in the length, are critical in cell development,

Correspondence to: Dr Feng-Yu Gao, Department of Gastroenterology, Hiser Medical Group of Qingdao, Medical College of Qingdao University, 5 Donghaizhong Road, Qingdao, Shandong 266033, P.R. China

E-mail: fengyu_gao@126.com

Key words: gastric cancer, retinoblastoma 1, microRNA-132, post-transcription proliferation, differentiation and apoptosis $(2,3)$. Previous studies have revealed that the dysregulation of miRs is tightly associated with tumorigenesis, including cell cycle progression, cell survival and angiogenesis (4,5). Thus, the aberrant expression of miRs may provide novel strategies for blocking the development of human cancers.

The expression and functions of miR-132 have been clearly elucidated in various types of cancer by previous studies. For example, miR-132 was found to be frequently downregulated in ductal carcinoma in situ of breast and acted as a tumor suppressor by inhibiting cell proliferation (6). Furthermore, miR-132 was shown to inhibit colorectal cancer cell invasion and metastasis via directly targeting zinc finger E-box binding homeobox 2 (ZEB2) (7). Notably, a recent study indicated that miR-132 expression levels in gastric cancer tissues were significantly higher than those in the corresponding normal tissues (8). The increased expression levels of miR-132 were associated with more frequent lymph node metastasis, more lymphatic tumor emboli and a more advanced stage (8). In addition, a functional variant at the miR-132 binding site in the CD80 gene was observed to alter the susceptibility to gastric cancer in a Chinese Han population (9). However, the precise roles and molecular mechanisms of miR-132 in the development of gastric cancer remain undefined. The aim of the present study was to investigate the expression and roles of miR-132 in the regulation of gastric cancer progression.

\section{Materials and methods}

Clinical tissue specimens. A total of 35 pairs of human gastric cancer and adjacent normal tissue specimens were obtained from routine therapeutic surgery at our department (Department of Gastroenterology, Medical College of Qingdao University, Qingdao, China) between May and November 2012. Informed consent was obtained from each patient prior to the collection of tissue samples. The present study was approved by the Ethics Committee of the Hiser Medical Group of Qingdao (Qingdao, China).

Cell culture and transient transfection. The gastric cancer cell lines (NCI-N87 and MGC80-3) were purchased from the Cell Bank of Type Culture Collection of Chinese Academy 
of Sciences (Shanghai, China). Cells were maintained at $37^{\circ} \mathrm{C}$ in an atmosphere of $5 \% \mathrm{CO}_{2}$ in Dulbecco's modified Eagle's medium (DMEM; Gibco Life Technologies, Beijing, China), supplemented with $10 \%$ fetal bovine serum, penicillin $(100 \mathrm{IU} / \mathrm{ml})$ and streptomycin $(100 \mathrm{mg} / \mathrm{ml}$; all Gibco Life Technologies). The miR-132 mimics and antisense oligos were purchased from Ambion Life Technologies (Carlsbad, CA, USA). All the transient transfections were performed using Lipofectamine 2000 (Invitrogen Life Technologies, Carlsbad, CA, USA) according to the manufacturer's instructions.

$R N A$ isolation and quantitative polymerase chain reaction $(q P C R)$. Total RNA was extracted using the mirVana ${ }^{\mathrm{TM}}$ miRNA Isolation kit (Ambion Life Technologies, Grand Island, NY, USA) according to the manufacturer's instructions and the expression of mature miRs was assayed using the TaqMan MicroRNA assay (Applied Biosystems, Shanghai, China). qPCR was performed using an Applied Biosystems 7300 Real-Time PCR system and a TaqMan Universal PCR Master Mix (Applied Biosystems Life Technologies, Foster City, CA, USA). The expression of miRs was normalized to that of U6 small nuclear RNA.

Western blot analysis. Human tissues or cells were harvested and lysed with ice-cold lysis buffer (pH 6.8-7.0). After centrifugation at $10,000 \mathrm{x}$ g for $10 \mathrm{~min}$ at $4^{\circ} \mathrm{C}$, the proteins in the supernatant were quantified and separated by $10 \%$ SDS-PAGE (Beyotime Institute of Biotechnology, Nantong, China) . The western blot assay was performed using anti-ZEB2 (cat. no ab25837; 1:1,000; rabbit anti-human polyclonal antibody; Abcam, Cambridge, MA, USA), anti-cyclin E1 (cat. no ab7959; 1:2,000; rabbit polyclonal antibody; Abcam), and anti-retinoblastoma 1 (RB1) antibodies (cat. no ab24; 1:1,000; mouse anti-human monoclonal antibody; Abcam). The protein levels were normalized to total GAPDH (cat. no. sc-365062; 1:5,000; mouse anti-human monoclonal antibody; Santa Cruz Biotechnology, Inc., Dallas, Texas, USA).

Cell proliferation and cell cycle analysis. The cell viability was determined by assaying the reduction of 3-(4,5-dimethylthiazol-2-yl)-2,5-diphenyltetrazolium bromide (MTT) to formazan. For bromodeoxyuridine (BrdU) analysis, a cell proliferation enzyme-linked immunosorbent assay (a BrdU kit; Beyotime Institute of Biotechnology, Shanghai, China) was used to analyze the incorporation of BrdU during DNA synthesis, and was conducted according to the manufacturer's instructions. For cell cycle analysis, the cells were labeled for 15 min with propidium iodide and immediately analyzed by flow cytometry (BD Biosciences, San Jose, CA, USA). The bar charts represent the percentage of cells in each phase of the cell cycle $\left(\mathrm{G}_{0} / \mathrm{G}_{1}, \mathrm{~S}\right.$ and $\left.\mathrm{G}_{2} / \mathrm{M}\right)$.

Luciferase reporter assays. The human RB1 gene 3'-untranslated region (UTR) was amplified by reverse-transcription PCR using cDNA from NCI-N87 cells. A 3'-UTR mutant construct was also generated by replacing the $3^{\prime}$-UTR with custom-made synthetic whole 3'-UTR DNAs with mismatched seed region mutations. miR-132 mimics or negative controls (NCs) and a pMIR-3'-UTR vector were co-transfected into the NCI-N87 cells. The NC was a scramble sequence (5'-TAC

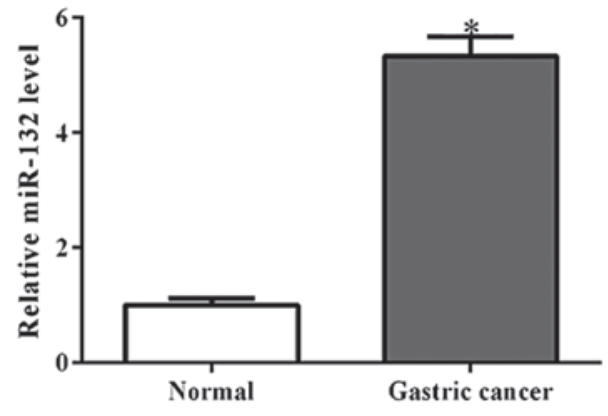

Figure 1. Expression level of miR-132 in gastric cancer tissues. miR-132 expression was determined by quantitative polymerase chain reaction in human gastric cancer tissues and adjacent normal tissues $(n=35) .{ }^{*} \mathrm{P}<0.001$. miR, microRNA.

GGATTGAACTGACTCGA-3'). Renilla and firefly luciferase activities were measured using a Dual-Luciferase Reporter system (Promega Corporation, Madison, WI, USA).

Statistical analysis. The data shown represent the mean \pm standard error values of at least three independent experiments. Comparisons between groups were analyzed using the t-test and $\chi^{2}$ test. $\mathrm{P}<0.05$ was considered to indicate a statistically significant difference.

\section{Results}

miR-132 expression is upregulated in gastric cancer tissues. Using qPCR analysis, the miR-132 expression levels were detected in 35 pairs of gastric cancer tissue and their matched adjacent non-cancerous tissues. As shown in Fig. 1, miR-132 was observed to be significantly increased in gastric cancer tissues $(\mathrm{P}<0.001)$. This finding is consistent with that of a previous report (8).

miR-132 promotes cell proliferation and invasion in vitro. To further determine the roles of miR-132 in tumorigenesis, its mimics or a NC were transfected into NCI-N87 cells (Fig. 2A). As expected, overexpression of miR-132 mimics promoted cell viability and proliferation, as shown by the MTT and BrdU incorporation assays (Fig. 2B and C). Cell cycle analysis also indicated that miR-132 overexpression led to a reduced percentage of cells in the $\mathrm{G}_{1} / \mathrm{G}_{0}$ phase and increased percentage of cells in the $\mathrm{S}$ phase, when compared with the NC-transfected cells (Fig. 2D; P<0.01). Additionally, the cell invasion abilities were observed to be increased by miR-132 mimics in the NCI-N87 cells (Fig. 2E). Similar results were observed in the MGC80-3 cells with miR-132 overexpression (Fig. 3A-E).

Next, the NCI-N87 and MGC80-3 cells were transfected with miR-132 antisense oligos, which block the functions of endogenous miR-203, to assess whether the inhibition of miR-132 inhibits cell growth. As expected, miR-132 antisense oligos inhibited the growth and invasion of gastric cancer cells, when compared with the NC-transfected cells (Fig. 4A-D; $\mathrm{P}<0.05)$. These data indicate that miR-132 may promote cell proliferation and invasion.

miR-132 directly targets RBI in gastric cancer cells. Previous studies have shown that various genes, such as 
A

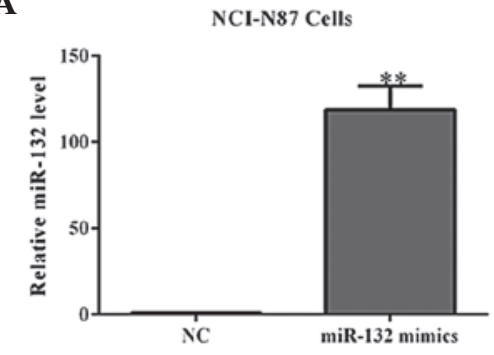

B

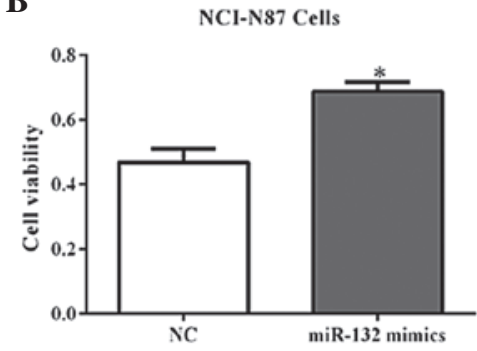

C

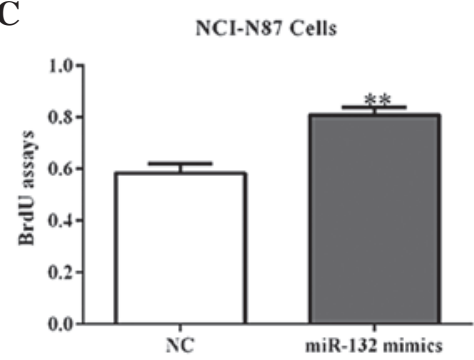

D

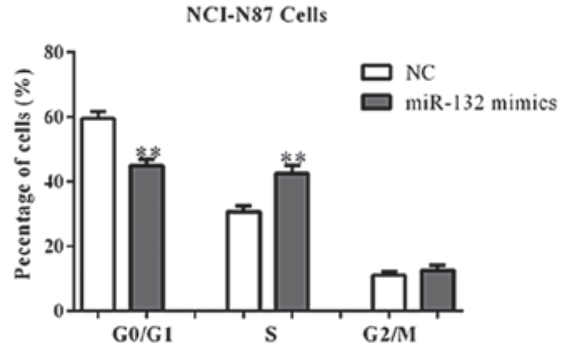

$\mathbf{E}$

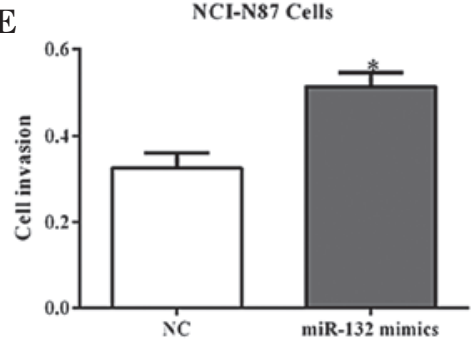

Figure 2. miR-132 mimics promote NCI-N87 cell proliferation, migration and invasion. (A) Expression levels of miR-132 following transfection of miR-132 mimics or the NC in NCI-N87 cells for $24 \mathrm{~h}$. (B) Cell viability and (C) proliferative potential were determined in NCI-N87 cells transfected with miR-132 mimics or the NC. (D) The NCI-N87 cells transfected with miR-132 mimics or the NC were analyzed by flow cytometry. (E) Cell invasion assays were performed in NCI-N87 cells transfected with miR-132 mimics or the NC. ${ }^{*} \mathrm{P}<0.05$ and ${ }^{* *} \mathrm{P}<0.01$ between the two groups. miR, microRNA; NC, negative control; BrdU, bromodeoxyuridine.

A

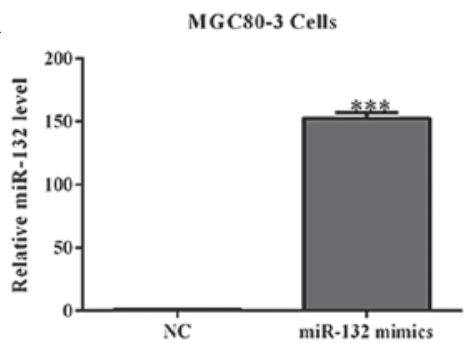

C

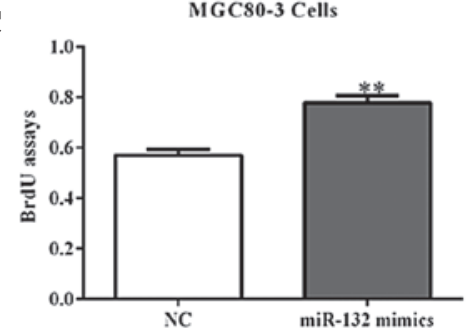

B

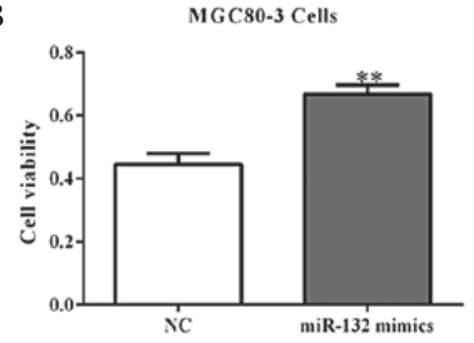

Figure 3. miR-132 mimics promote MGC80-3 cell proliferation, migration and invasion. (A) Expression levels of miR-132 following transfection of miR-132 mimics or the NC in MGC80-3 cells for $24 \mathrm{~h}$. (B) Cell viability and (C) proliferative potential were determined in MGC80-3 cells transfected with miR-132 mimics or the NC. (D) The MGC80-3 cells transfected with miR-132 mimics or the NC were analyzed by flow cytometry. (E) Cell invasion assays were performed in MGC80-3 cells transfected with miR-132 mimics or the $\mathrm{NC}$. ${ }^{*} \mathrm{P}<0.05,{ }^{* *} \mathrm{P}<0.01$ and ${ }^{* * *} \mathrm{P}<0.001$ between the two groups. miR, microRNA; $\mathrm{NC}$, negative control; BrdU, bromodeoxyuridine.

ZEB2 and cyclin E1 are negatively regulated by miR-132 in human cancer cells $(7,10)$. However, in the present study, no changes were detected in the protein contents of ZEB2 and cyclin E1 in NCI-N87 cells transfected with miR-132 mimics or antisense oligos (Fig. 5A-B). Therefore, a TargetScan algorithm (http://www.targetscan.org) was used to facilitate the identification of miR-132 targets in human gastric cancer cells. Among which, it was found that the RB1 gene, a tumor suppressor, harbored a potential miR-132 binding site in its 3'-UTR (Fig. 5C). Therefore, the full-length RB1 3'-UTR was 
A

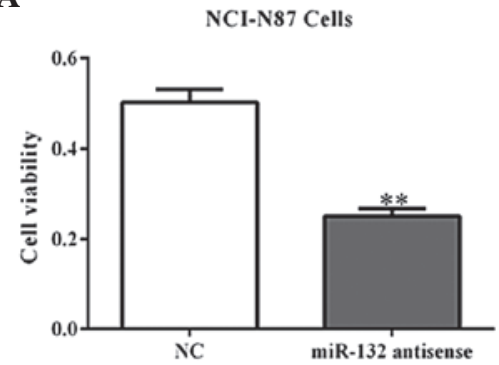

C

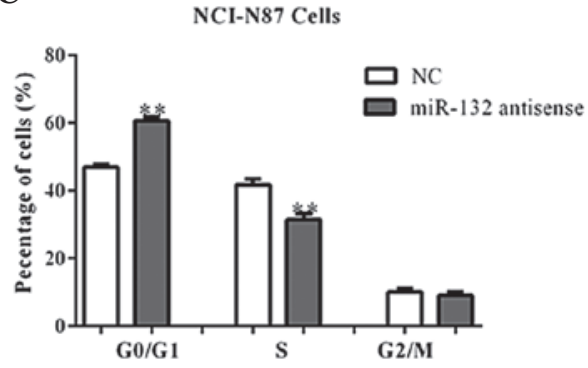

B

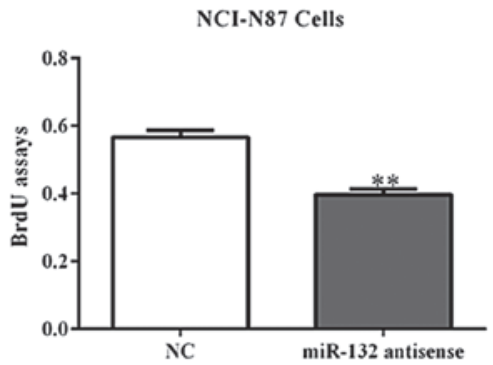

D

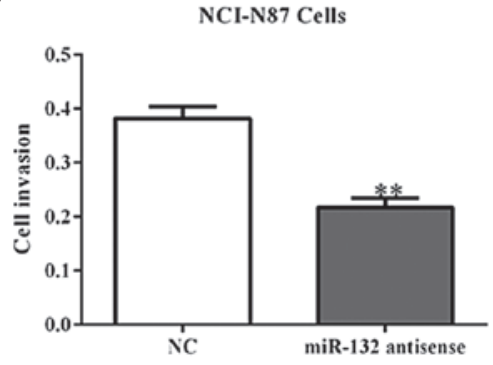

Figure 4. miR-132 antisense oligos inhibit gastric cancer cell proliferation, migration and invasion. (A) Cell viability and (B) proliferative potential were determined in NCI-N87 cells transfected with miR-132 antisense oligos or the NC for $24 \mathrm{~h}$. (C) The cell cycle phase of NCI-N87 cells transfected with miR-132 antisense oligos or the NC were analyzed by flow cytometry. (D) Cell invasion assays were performed in NCI-N87 cells transfected with miR-132 antisense oligos or the NC. ${ }^{* *} \mathrm{P}<0.01$ between the two groups. miR, microRNA; NC, negative control; BrdU, bromodeoxyuridine.

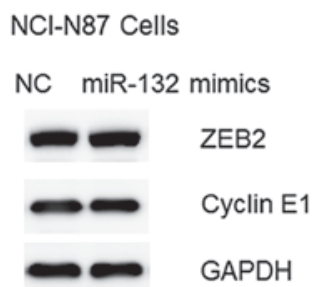

B
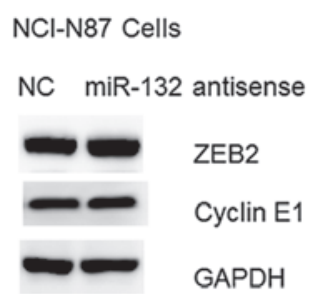

C

\begin{tabular}{ll}
\multicolumn{1}{c}{ MicroRNA } & \multicolumn{1}{c}{ Gene } \\
\hline has-miR-132 & CFL2 \\
has-miR-132 & SLC26A7 \\
has-miR-132 & LAMC2 \\
has-miR-132 & RAD21 \\
has-miR-132 & SOX11 \\
has-miR-132 & RB1 \\
\hline
\end{tabular}

D

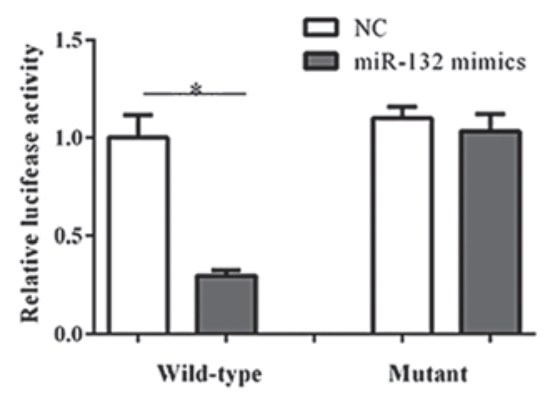

F

G

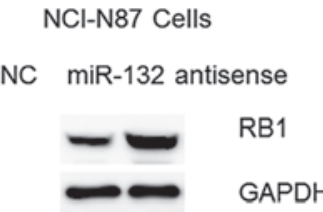

Patient 1

Normal Gastric cancer

RB1

GAPDH
NCI-N87 Cells

NC miR-204 mimics

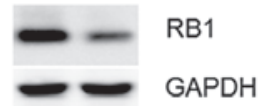

Patient 2

Normal Gastric cancer

Figure 5. miR-132 negatively regulates RB1 expression in gastric cancer cells. (A and B) Western blot analysis of ZEB2 and cyclin E1 expression in NCI-N87 cells transfected with (A) miR-132 mimics, (B) miR-132 antisense oligos or the NC. (C) Computer (TargetScan) prediction of miR-132 binding sites in the 3'-UTRs of various target genes. (D) Luciferase reporter assays in NCI-N87 cells. Cells were transfected with 100 ng wild-type 3'-UTR-reporter or mutant constructs together with miR-132 mimics or the NC. (E) Western blot analysis of RB1 expression in NCI-N87 cells transfected with miR-132 mimics or the NC. (F) Western blot analysis of RB1 expression in NCI-N87 cells transfected with miR-132 antisense oligos or the NC. (G) Representative protein levels of RB1 were analyzed by western blot analysis of gastric cancer or normal tissue samples from two patients." $\mathrm{P}<0.01$ between the two groups. miR, microRNA; $\mathrm{NC}$, negative control; RB1, retinoblastoma 1; ZEB2, zinc finger E-box binding homeobox 2; UTR, untranslated region. 
A

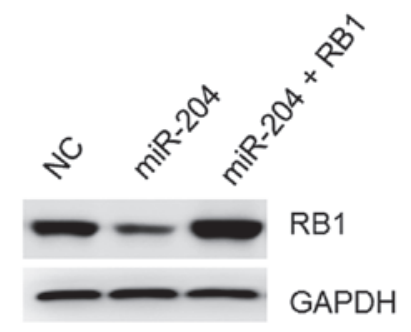

C

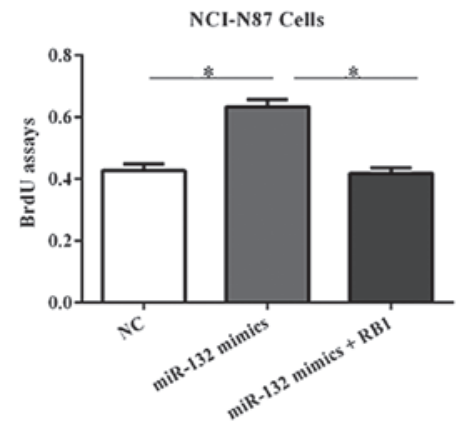

B

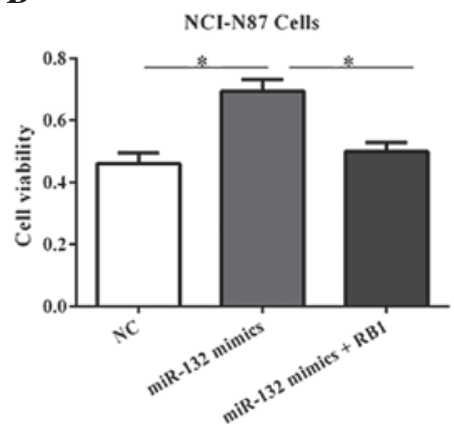

D

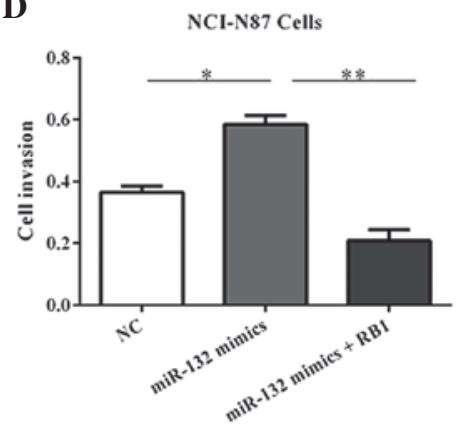

Figure 6. RB1 reintroduction reverses the proliferative roles of miR-132. (A) RB1 protein expression was determined by western blot analysis in NCI-N87 cells. Cells were pre-transfected with miR-132 mimics or the NC for $24 \mathrm{~h}$, and subsequently transfected with expression plasmids for RB1 or an empty vector for a further $24 \mathrm{~h}$. (B) Cell viability, (C) proliferation and (D) invasion abilities were determined in the NCI-N87 cells. ${ }^{*} \mathrm{P}<0.05$ and ${ }^{* *} \mathrm{P}<0.01$ between the two groups. miR, microRNA; RB1, retinoblastoma 1; NC, negative control; BrdU, bromodeoxyuridine.

cloned into a luciferase reporter vector. Overexpression of the miR-132 mimics reduced the luciferase activity using the wild-type RB1 3'-UTR (Fig. 5D). However, mutation of the putative miR-132 binding site abrogated the luciferase responsiveness to miR-132 (Fig. 5D). Furthermore, transfection of miR-132 mimics in NCI-N87 cells resulted in a reduced RB1 protein abundance (Fig. 5E). In accordance with this, a marked increase in RB1 content was observed in the cells with miR-132 inhibition (Fig. 5F). Furthermore, the RB1 protein content was observed to be decreased in the gastric cancer tissue samples (Fig. 5G), which correlated with the upregulation of miR-132 in the tumor tissue samples (Fig. 1). Taken together, these results indicate that RB1 may be a downstream target of miR-132 in gastric cancer cells.

RB1 overexpression blocks the proliferative role of miR-132. To verify the functional connection between miR-132 and $\mathrm{RB} 1$, the NCI-N87 cells were transfected with RB1 expression plasmids or an empty vector following transfection of miR-132 mimics (Fig. 6A). As shown in Fig. 6B-D, RB1 reintroduction reversed the oncogenic roles of miR-132, underlining the specific importance of RB1 for miR-132 action in tumorigenesis.

\section{Discussion}

Numerous studies have demonstrated that various miRs, such as miR-375, -200 and -516a-3p are dysregulated in gastric cancer tissues or cell lines (11-13). In addition, abnormal expression of certain miRs in gastric cancer is associated with progression, poor prognosis and sensitivity to chemotherapy $(14,15)$. Therefore, identification and understanding of the roles of miRs will expand their application in clinical practice, to be used in the detection, diagnosis, prognosis and treatment of gastric cancer.

Previous studies have shown that miR-132 is downregulated and may serve as a tumor suppressor in prostate, lung and breast cancer (16-18). In the present study, evidence demonstrating that miR-132 is upregulated in gastric cancer tissues is presented. In addition, in vitro studies further indicated that the overexpression of miR-132 mimics promoted (while its antisense oligos suppressed) cell proliferation and invasion. Although the reason why miR-132 could either inhibit or promote cell proliferation remains unknown, the role of miR-132 in tumorigenesis may be cell or tissue-specific. However, the functions of miR-132 in gastric cancer development require further investigation in vivo; thus, analysis in miR-132 knockout mice or overexpression of miR-132 in nude mice may be facilitative.

At the molecular level, computer-assisted analysis with TargetScan revealed that RB1 was a direct target of miR-132 in gastric cells. RB1 is the first tumor suppressor gene that was identified and is a negative regulator of the cell cycle (19). It has been shown that RB1 expression is inhibited by various miRs in human cancer cells (20-22), indicating that downregulation of RB1 may be a key feature of tumor initiation or progression.

In conclusion, miR-132 was identified as a novel positive regulator of gastric cancer development via suppression of RB1 protein expression. These data may be beneficial for determining novel therapeutic strategies for future treatment of gastric cancer. 


\section{References}

1. Shen L, Shan YS, Hu HM, Price TJ, Sirohi B, Yeh KH, Yang YH, Sano T, Yang HK, Zhang X, et al: Management of gastric cancer in Asia: Resource-stratified guidelines. Lancet Oncol 14: e 535-e547, 2013.

2. Sun K and Lai EC: Adult-specific functions of animal microRNAs. Nat Rev Genet 14: 535-548, 2013.

3. Ameres SL and Zamore PD: Diversifying microRNA sequence and function. Nat Rev Mol Cell Biol 14: 475-488, 2013.

4. Ling H, Fabbri M and Calin GA: MicroRNAs and other non-coding RNAs as targets for anticancer drug development. Nat Rev Drug Discov 12: 847-865, 2013.

5. Song S and Ajani JA: The role of microRNAs in cancers of the upper gastrointestinal tract. Nat Rev Gastroenterol Hepatol 10: 109-118, 2013.

6. Li S, Meng H, Zhou F, Zhai L, Zhang L, Gu F, Fan Y, Lang R, $\mathrm{Fu} \mathrm{L}, \mathrm{Gu} \mathrm{L}$, et al: MicroRNA-132 is frequently down-regulated in ductal carcinoma in situ (DCIS) of breast and acts as a tumor suppressor by inhibiting cell proliferation. Pathol Res Pract 209: $179-183,2013$

7. Zheng YB, Luo HP, Shi Q, Hao ZN, Ding Y, Wang QS, Li SB, Xiao GC and Tong SL: MiR-132 inhibits colorectal cancer invasion and metastasis via directly targeting ZEB2. World J Gastroenterol 20: 6515-6522, 2014.

8. Liu X, Yu H, Cai H and Wang Y: The expression and clinical significance of miR-132 in gastric cancer patients. Diagn Pathol 9: 57, 2014.

9. Wu R, Li F, Zhu J, Tang R, Qi Q, Zhou X, Li R, Wang W, Hua D and Chen W: A functional variant at miR-132-3p, miR-212-3p, and miR-361-5p binding site in CD80 gene alters susceptibility to gastric cancer in a Chinese Han population. Med Oncol 31: 60, 2014.

10. Wang J, Xu G, Shen F and Kang Y: MiR-132 targeting cyclin E1 suppresses cell proliferation in osteosarcoma cells. Tumour Biol 35: 4859-4865, 2014

11. Tsukamoto Y, Nakada C, Noguchi T, Tanigawa M, Nguyen LT, Uchida T, Hijiya N, Matsuura K, Fujioka T, Seto M, et al: MicroRNA-375 is downregulated in gastric carcinomas and regulates cell survival by targeting PDK1 and 14-3-3zeta. Cancer Res 70: 2339-2349, 2010.
12. Shinozaki A, Sakatani T, Ushiku T, Hino R, Isogai M, Ishikawa S, Uozaki H, Takada K and Fukayama M: Downregulation of microRNA-200 in EBV-associated gastric carcinoma. Cancer Res 70: 4719-4727, 2010.

13. Takei Y, Takigahira M, Mihara K, Tarumi Y and Yanagihara K: The metastasis-associated microRNA miR-516a-3p is a novel therapeutic target for inhibiting peritoneal dissemination of human scirrhous gastric cancer. Cancer Res 71: 1442-1453, 2011.

14. Shin VY and Chu KM: MiRNA as potential biomarkers and therapeutic targets for gastric cancer. World J Gastroenterol 20: 10432-10439, 2014.

15. Ma J, Hong L, Chen Z, Nie Y and Fan D: Epigenetic regulation of microRNAs in gastric cancer. Dig Dis Sci 59: 716-723, 2014.

16. Formosa A, Lena AM, Markert EK, Cortelli S, Miano R, Mauriello A, Croce N, Vandesompele J, Mestdagh P, Finazzi-Agrò E, et al: DNA methylation silences miR-132 in prostate cancer. Oncogene 32: 127-134, 2013.

17. You J, Li Y, Fang N, Liu B, Zu L, Chang R, Li X and Zhou Q: MiR-132 suppresses the migration and invasion of lung cancer cells via targeting the EMT regulator ZEB2. PLoS One 9: e91827, 2014.

18. Tahiri A, Leivonen SK, Luders T, Steinfeld I, Ragle Aure M, Geisler J, Mäkelä R, Nord S, Riis ML, Yakhini Z, et al: Deregulation of cancer-related miRNAs is a common event in both benign and malignant human breast tumors. Carcinogenesis 35: 76-85, 2014.

19. Chen HZ, Tsai SY and Leone G: Emerging roles of E2Fs in cancer: An exit from cell cycle control. Nat Rev Cancer 9: 785-797, 2009.

20. Deng Y, Huang Z, Xu Y, Jin J, Zhuo W, Zhang C, Zhang X, Shen M, Yan X, Wang L, et al: MiR-215 modulates gastric cancer cell proliferation by targeting RB1. Cancer Lett 342: 27-35, 2014.

21. Zhang YF, Zhang AR, Zhang BC, Rao ZG, Gao JF, Lv MH, Wu YY, Wang SM, Wang RQ and Fang DC: MiR-26a regulates cell cycle and anoikis of human esophageal adenocarcinoma cells through Rb1-E2F1 signaling pathway. Mol Biol Rep 40: 1711-1720, 2013.

22. Feng S, Cong S, Zhang X, Bao X, Wang W, Li H, Wang Z, Wang G, Xu J, Du B, et al: MicroRNA-192 targeting retinoblastoma 1 inhibits cell proliferation and induces cell apoptosis in lung cancer cells. Nucleic Acids Res 39: 6669-6678, 2011. 